\title{
Optimal Oil-Owner Behavior in Piecewise Deterministic Models
}

\author{
Atle Seierstad \\ Department of Economics, University of Oslo, P.O. Box 1095, Blindern, 0317 Oslo, Norway
}

Correspondence should be addressed to Atle Seierstad; atle.seierstad@econ.uio.no

Received 29 April 2013; Revised 23 August 2013; Accepted 18 September 2013

Academic Editor: Alain Piétrus

Copyright (C) 2013 Atle Seierstad. This is an open access article distributed under the Creative Commons Attribution License, which permits unrestricted use, distribution, and reproduction in any medium, provided the original work is properly cited.

Six simple piecewise deterministic oil production models from economics are discussed by using solution tools that are available in the theory of piecewise deterministic optimal control problems.

\section{Introduction}

Six simple piecewise deterministic models for optimal oilowner behavior are presented. Their central property is sudden jumps in states. The aim of this paper is to show in admittedly exceedingly simple models how available tools for piecewise deterministic models, namely, the HJB equation and the maximum principle, can be used to solve these models analytically. We are looking for solutions given by explicit formulas. That can only be obtained if the models are simple enough. The models may be too simple to be of much interest in themselves, but they can provide some intuition about features optimal solutions may have in more complicated models.

Piecewise deterministic models have been used a number of times in economic problems in the literature; some few scattered references are given that contain such applications [1-4]. I have not been able to find references directly concerned with piecewise deterministic oil production problems. For different probability structures, and for discrete time, a host of related problems has been discussed in the literature; references to such literature have been left out, with one exception. Problems of control of jump diffusions, see [5], encompass piecewise deterministic problems, and some problems appearing in [5] are related to the ones discussed below. A classic reference to piecewise deterministic control problems is [2].

In all models below, an unbounded number of jumps in the state can occur at times $0<\tau_{1}<\tau_{2}<\tau_{3}<\cdots$, and, when $\tau_{j}$ is given, $\tau_{j+1}$ is exponentially distributed in $\left[\tau_{j}, \infty\right)$ (all $\tau_{i}-\tau_{i-1}$ independent). Sometimes, the size of the jumps is influenced by stochastic variables $V_{j}$. Let $\omega=\left(\tau_{1}, V_{1}, \tau_{2}, V_{2}, \ldots\right)$. At time $t$, we imagine that the control values chosen can be allowed to depend on what has happened, that is, on $\tau_{i}, V_{i}$ for $\tau_{i}<t$, but not on future events, that is, $\tau_{i}, V_{i}$ for which $\tau_{i}>t$. Such controls (written $u(t, \omega)$ ) are called nonanticipative. Corresponding state solutions denoted by $x(t, \omega)$ are then also nonanticipative. (A general set-up, with further explanations, is given in Appendix.) Frequently below, $x(t, \omega)$ will be the wealth of the oil owner. In infinite horizon economic models, the weakest terminal condition that is natural to apply is an expectational no-Ponzigame condition; namely, $\liminf _{T \rightarrow \infty} E\left[e^{-k T} x(T, \omega)\right] \geq 0$, where $k$ is the discount factor. Note that some stronger conditions will be used in some of the models presented in the sequel.

\section{Model 1}

Consider the optimization problem

$$
\begin{aligned}
& \max _{u(\cdot)} E\left[\int_{0}^{T} \gamma^{-1} e^{-\delta t} u^{\gamma} d t+a x(T)\right] \\
& a, \delta>0, \quad \gamma \in(0,1), \quad a, \delta, \gamma \text { fixed, }
\end{aligned}
$$

where $u$ is the control, subject to the state dynamics,

$$
\dot{x}=k x-u, \quad u \geq 0, x(0)=x_{0}>0,
$$

where $k$ and $x_{0}$ are fixed positive constants,

$$
x\left(\tau_{i}+\right)-x\left(\tau_{i}^{-}\right)=\alpha_{i}, \quad \alpha_{i}>0, \quad \sum \alpha_{i}<\infty,
$$


$\alpha_{i}$ fixed. (Formally, only $\sup _{i} \alpha_{i}<\infty$ is needed.) Let $\tau_{0}=0$. There is a fixed intensity $\lambda$ of jumps; that is, for $i=1,2, \ldots$, given $\tau_{i-1}, \tau_{i} \sim \lambda e^{-\lambda\left(\tau_{i}-\tau_{i-1}\right)}, \tau_{i} \geq \tau_{i-1}$ (all $\tau_{i}-\tau_{i-1}$ independent), and we assume $\delta>k$.

The interpretation of the model is that $u$ is consumption rate, $\alpha_{i}$ is the size (in dollars) of an oil field found at time $\tau_{i}$, $x$ is wealth, and $k x$ is interest. Oil fields are sold immediately after discovery.

Let us solve problem (1)-(3) by using the extremal method (see the appendix). Now, with $H=e^{-\delta t} u^{\gamma} / \gamma+p(k x-$ $u$ ), solving the first-order condition for maximum of $H$, we get that $u=e^{\delta t /(\gamma-1)} p^{1 /(\gamma-1)}$ maximizes the Hamiltonian.

The adjoint equation (see (A.8) in Appendix) is

$$
\dot{p}(t, j)=-k p(t, j)+\lambda p(t, j)-\lambda p(t, j+1), \quad p(T)=a ;
$$

we guess that we do not need the fact that $p(t, j)$ depends on arbitrary initial points $(s, y)$, as in appendix. Equation (4) is satisfied by $p(t)=\widehat{a} e^{-k t}, \widehat{a}:=a e^{k T}$ (we try the possibility that $p(t, j)$ is even independent of $j)$.

Now, for $b:=\widehat{a}^{1 /(\gamma-1)}, \rho:=(k-\delta) /(\gamma-1)>0$, we get $u(t)=$ $e^{\delta t /(\gamma-1)}(p(t))^{1 /(\gamma-1)}=b e^{-\rho t}$. Because $H$ is concave in $(x, u)$, $a x$ is linear, and $\alpha_{i}$ is independent of $x$, sufficient conditions based on concavity (see Theorem 3 in Appendix) then give us that the control $u=b e^{-\rho t}$ is an optimal control for problem (1)-(3). The optimal control is independent of $\lambda$ and the $\alpha_{i}$ 's.

Write $\beta:=b /(k+\rho)$. For $u=b e^{-\rho t}$, we have that $\dot{x}^{*}=k x^{*}-b e^{-\rho t}$. The solution of this equation equals, for $t \in\left(\tau_{i}, \tau_{i+1}\right)$,

$$
x^{*}(t)=\beta e^{-\rho t}+\left(x_{0}-\beta\right) e^{k t}+\sum_{j=1}^{i} \alpha_{j} e^{k\left(t-\tau_{j}\right)},
$$

To see this, find $\dot{x}^{*}(t)$ and simply check that the differential equation is satisfied. Moreover, evidently $x^{*}(\cdot)$ satisfies $x^{*}\left(\tau_{i}+\right)=x^{*}\left(\tau_{i}-\right)+\alpha_{i}$. (To ensure that $x^{*}(t) \geq 0$ a.s., we could have postulated at the outset that $a$ is so large that $x_{0} \geq \beta$.)

Write $x^{*}(t)=e^{k t} \Phi_{i}(t, \omega)$, for $t \in\left(\tau_{i}, \tau_{i+1}\right)$, where $\Phi_{i}(t, \omega)=\left(e^{-(\rho+k) t}-1\right) \beta+\sum_{j=1}^{i} \alpha_{j} e^{-k \tau_{j}}+x_{0}$.

We are now going to replace the bequest function $a x(T)$ by the terminal constraint $\operatorname{Ex}(T, \omega) \geq 0$. For this purpose, we are now going to vary $a$ and hence also $\widehat{a}$ and $\beta$. For $t=T$, consider the expectation of $\Phi_{i}(t, \omega)$. We will not give an explicit formula for this expectation, but we mention that it can be calculated in two steps, first given that $i$ jumps have happened in $[0, T]$ and then the expectation with $i$ being stochastic (the rule of double expectations is then used). The expectation of the sum containing $\alpha_{j}$ as well as $x_{0}$ is positive, and the term in front of $\beta$ is negative. There thus exists a unique positive value $\beta_{T}$ of $\beta$ and, hence, of $a$ (denoted by $\left.a_{T}\right)$ such that $E \Phi_{i}(T, \omega)=0$; that is, $E x^{*}(T, \omega)=0$.

If we drop the term $a x(T)$ in the criterion but add the terminal condition $\operatorname{Ex}(T, \omega) \geq 0$, the free end optimality obtained in the original problem for $a=a_{T}$ evidently means optimality of $u=b e^{-\rho t}=\widehat{a}_{T}^{1 /(\gamma-1)} e^{-\rho t}=\left(a_{T} e^{k T}\right)^{1 /(\gamma-1)} e^{-\rho t}$ in the end constrained problem (the found value $a_{T}$ appears in $u$, though now not in the criterion). (Alternatively, we could use the sufficient conditions for end constrained problems in Theorem 3 in Appendix to obtain optimality in the present end constrained problem. This would require us to check condition (A.13), which is easily done.)

Let us discuss a little more the value of $\beta$ for large values of $T$. Now,

$$
\begin{aligned}
E\left[e^{-k \tau_{j}}\right] & =E\left[E\left[e^{-k \tau_{j}} \mid \tau_{j-1}\right]\right]=E\left[e^{-k \tau_{j-1}} \int_{0}^{\infty} \lambda e^{-k x} e^{-\lambda x} d x\right] \\
& =E E\left[\left[\frac{\lambda}{k+\lambda}\right] e^{-k \tau_{j-1}} \mid \tau_{j-2}\right] \\
& =E\left[\left[\frac{\lambda}{k+\lambda}\right]^{2} e^{-k \tau_{j-2}}\right] .
\end{aligned}
$$

And, continuing, we get $E\left[e^{-k \tau_{j}}\right]=[\lambda /(k+\lambda)]^{j}$.

Then, $E \Phi_{i}(\infty, \omega)=x_{0}+\sum_{j=1}^{i} \alpha_{j}[\lambda /(k+\lambda)]^{j}-\beta$.

Now, with probability 1 an infinite number of jumps occur in $[0, \infty)$, and

$$
E \Phi_{\infty}(\infty, \omega)=x_{0}+\sum_{j=1}^{\infty} \alpha_{j}\left[\frac{\lambda}{k+\lambda}\right]^{j}-\beta
$$

Denote by $\beta_{\infty}$ the value of $\beta$ for which $E \Phi_{\infty}(\infty, \omega)=0$, and define $b_{\infty}:=\beta_{\infty}(\rho+k)$. For $T$ being large, $E \Phi_{i}(T, \omega) \approx$ $E \Phi_{i}(\infty, \omega)=E \Phi_{\infty}(\infty, \omega)$, so $\beta_{T}$ is close to $\beta_{\infty}$ and $b_{T}:=$ $\beta_{T}(\rho+k)$ is close to $b_{\infty}$. So for $T$ being large, the optimal control is approximately $b_{\infty} e^{-\rho t}$. Note that $\rho$ belongs to $(\delta-$ $k, \infty)$ and both $\rho$ and $b_{\infty}$ are increasing in $\gamma \in(0,1)$, so a larger $\gamma$ means we consume more in the beginning. In the problem where $a x(T)$ is replaced by the terminal constraint $\operatorname{Ex}(T, \omega) \geq 0$, the optimal control depends on $\alpha_{i}, \lambda$. It is immediately seen that $\beta_{\infty}$ is increasing in $\lambda$ and in each $\alpha_{i}$, so also $b_{\infty}$ has these properties, indicating, for $T$ being large, that even $\beta_{T}$ and $b_{T}$ have these properties. This conclusion actually follows for any $T$, because $E\left[\sum_{j=1}^{i} \alpha_{j} e^{-k \tau_{j}}\right]$ is evidently increasing in $\lambda$ and in each $\alpha_{i}$.

We may assume that the jumps are stochastic, that is, that $x\left(\tau_{i}+\right)-x\left(\tau_{i}-\right)=V_{i}$, where $\left\{V_{i}\right\}_{i}$ take values in a common bounded set and are independent, and are independent of the $\tau_{i}$ 's. If we then assume that $\sum E\left(V_{i}\right)<\infty$, the solution in this problem is the same as the one above for $\alpha_{i}=E\left(V_{i}\right)$.

Note that we must assume that we have a deal with the bank in which our wealth is placed that it accepts the above behavior. That is, before time 0 , we have got an acceptance for the possibility of operating with this type of admissible solutions, which means that only in expectation we leave a wealth in the bank $\geq 0$. In actual runs, sometimes we leave in the bank a positive wealth (that the bank gets), and for other runs a negative wealth (debt) that the bank has to cover.

Consider now the case where $T=\infty, a=0$ in (1). Then, for $\widehat{a}=\left[\beta_{\infty}(\rho+k)\right]^{\gamma-1}$,

$$
\begin{aligned}
\lim _{T \rightarrow \infty} E\left[p(T) x^{*}(T, \omega)\right] & =\lim _{T \rightarrow \infty} E \widehat{a} \Phi_{i}(T, \omega) \\
& =\widehat{a} E \Phi_{\infty}(\infty, \omega)=0,
\end{aligned}
$$


when $\beta=\beta_{\infty}$. In this case, $u=b_{\infty} e^{-\rho t}$ is optimal in the problem where $\lim \inf _{T \rightarrow \infty} E[p(T) x(T, \omega)] \geq 0$ is added as an end constraint. (See the appendix, (A.14).) The latter condition is equivalent to the so-called no-Ponzi-game condition.

\section{Model 2}

(This model Is related to exercise 4.1 in Øksendal and Sulem [5].)

Consider the following problem:

$\max _{u(\cdot)} \int_{0}^{\infty} e^{-\delta t} u^{\gamma} d t, \quad$ where $\delta, \gamma$ are fixed positive constants

$$
\gamma \in(0,1), u \geq 0
$$

where $u$ is the control, subject to

$$
\begin{gathered}
\dot{x}=k x-u, \quad k, x_{0} \text { are given positive numbers, } \\
x\left(\tau_{i}+\right)-x\left(\tau_{i}-\right)=\alpha x\left(\tau_{i}-\right), \quad x(t)>0 \forall t, x(0)=x_{0},
\end{gathered}
$$

where $\alpha$ is a given positive number, $\delta>\gamma k, \tau_{i}, i=1,2, \ldots$, is exponentially distributed in $\left(\tau_{i-1}, \infty\right)$ with intensity $\lambda_{i}$ and $\lambda_{i}$ is decreasing towards zero $\left(\tau_{0}=0\right.$, all $\tau_{i}-\tau_{i-1}$ independent).

In contrast to problem (1)-(3), now the jumps are linearly dependent on $x\left(\tau_{i}-\right)$. To defend such a feature, one might argue that the richer we are, the more we are able to generate large jumps (the jump may actually represent a collection of oil finds). On the other side, we will assume that such jumps occur with smaller and smaller intensities.

It is easy to see that the current value function $\widetilde{J}(y, j)$ is of the form $\widetilde{A}_{j} y^{\gamma}$ when we replace $x_{0}$ by an arbitrary start value $y$. For any pair $(x(t, \omega), u(t, \omega))$ satisfying $(10)$, we can write $u(t, \omega)=v(t, \omega) x(t, \omega), v(t, \omega) \geq 0$. Then, for $t \in\left(\tau_{i}, \tau_{i+1}\right)$,

$$
\begin{aligned}
x(t, \omega) & =(1+\alpha) x\left(\tau_{i}-\right) e^{k\left(t-\tau_{i}\right)-\int_{\tau_{i}}^{t} v(s, \omega) d s} \\
& =(1+\alpha)^{2} x\left(\tau_{i-1}-\right) e^{k\left(t-\tau_{i-1}\right)-\int_{\tau_{i-1}}^{t} v(s, \omega) d s} \\
& =\cdots(1+\alpha)^{i} y e^{k t-\int_{0}^{t} v(s, \omega) d s},
\end{aligned}
$$

so $u(t)=y$ times some function $w(t, \omega), t \in\left(\tau_{i}, \tau_{i+1}\right)$. Then, the value of the criterion for this $v(\cdot)$, denoted by $\widehat{J}(y, v(\cdot)$,$) , is$ proportional to $y^{\gamma}$ and so is also $\sup _{v(\cdot,)} \widehat{J}(y, v(\cdot)$,$) . A similar$ argument works for $\widetilde{J}(y, j)$, the optimal value of the criterion when $j \geq 1$ jumps have already occurred at time $t=0$. So $\widetilde{J}(y, j)=\widetilde{A}_{j} y^{\gamma}, j=0,1, \ldots$ Writing $J$ and $A_{j}$ instead of $\widetilde{J}$ and $\widetilde{A}_{j}$, the current value function $J$ satisfies the following HJB equation:

$$
\begin{aligned}
\delta J(x, j)= & \max _{u}\left\{u^{\gamma}+J_{x}(x, j)(k x-u)\right\} \\
& -\lambda_{i+1}(J(x, j)-J((1+\alpha) x, j+1)) \\
= & \max _{u}\left\{u^{\gamma}+\gamma A_{j} x^{\gamma-1}(k x-u)\right\} \\
& -\lambda_{i+1}\left(A_{j} x^{\gamma}-(1+\alpha)^{\gamma} A_{j+1} x^{\gamma}\right) .
\end{aligned}
$$

The first order condition for maximum is $\gamma u^{\gamma-1}-\gamma A_{j} x^{\gamma-1}=0$. So $u=B_{j} x$, for $B_{j}=\left(A_{j}\right)^{1 /(\gamma-1)}$. Then,

$$
\begin{aligned}
\delta A_{j} x^{\gamma}= & B_{j}^{\gamma} x^{\gamma}+\gamma k A_{j} x^{\gamma}-\gamma A_{j} x^{\gamma} B_{j} \\
& -\lambda_{i+1}\left(A_{j} x^{\gamma}-(1+\alpha)^{\gamma} A_{j+1} x^{\gamma}\right),
\end{aligned}
$$

or dividing by $x^{\gamma}$ and rearranging,

$$
\begin{aligned}
0= & -\delta A_{j}+A_{j}^{\gamma /(\gamma-1)}(1-\gamma)+\gamma k A_{j} \\
& -\lambda_{j+1} A_{j}+\lambda_{j+1}(1+\alpha)^{\gamma} A_{j+1} .
\end{aligned}
$$

Dividing by $A_{j}$ gives

$$
0=-\delta+\gamma k-\lambda_{j+1}+\frac{\lambda_{j+1}(1+\alpha)^{\gamma} A_{j+1}}{A_{j}}+A_{j}^{1 /(\gamma-1)}(1-\gamma) .
$$

Let $A_{(\lambda)}$ satisfy

$$
\delta-\gamma k+\lambda-\lambda(1+\alpha)^{\gamma}=A_{(\lambda)}^{1 /(\gamma-1)}(1-\gamma)
$$

Evidently, relationship (16) would be obtained from (15) if all $\lambda_{j}=\lambda\left(\Rightarrow A_{j}=A_{j+1}\right)$; in that case, the optimal value function would be $A_{(\lambda)} y^{\gamma}$.

Now, assume first, for some $k^{*}$, that $\lambda_{k^{*}+1}=\lambda_{k^{*}+2}=\cdots=$ 0 . Then, $A_{k^{*}} y^{\gamma}$ is the optimal value in the problem with no jumps, so $A_{k^{*}}=A_{(0)}$. Given $A_{k^{*}}$, (14) determines $A_{k^{*}-1}$, $A_{k^{*}-2}, \ldots, A_{0}$. It must be the case that $A_{j}$ is decreasing. Given the same start point $x$, the optimal value when $j+1$ jumps have occurred already at time 0 must be smaller than the optimal value when $j$ jumps have occurred at time 0 ; in the former problem, prospective jumps have smaller probabilities for happening. (Let us show by backwards induction that $A_{j}$, $j=0,1,2, \ldots, k$, is nonincreasing. Evidently, $A_{k}=A_{k+1}$. Assume by induction that $A_{j} \geq A_{j+1}$. If $A_{j-1}<A_{j}$, then, by (15), $(\gamma-1)\left(A_{j-1}\right)^{1 /(\gamma-1)}=-\delta+\gamma k-\lambda_{j}+\lambda_{j}(1+\alpha)^{\gamma} A_{j} / A_{j-1}>$ $-\delta+\gamma k-\lambda_{j+1}+\lambda_{j+1}(1+\alpha)^{\gamma} A_{j} / A_{j-1}>-\delta+\gamma r-\lambda_{j+1}+$ $\lambda_{j+1}(1+\alpha)^{\gamma} A_{j+1} / A_{j}=(\gamma-1)\left(A_{j}\right)^{1 /(\gamma-1)}$. Because $\gamma-$ $1<0$ and $z \rightarrow(z)^{1 /(\gamma-1)}$ is decreasing, a contradiction is obtained.) As we will let $k^{*}$ vary, denote the sequence $A_{0}, \ldots, A_{k^{*}}$ defined by (14) for $A_{k^{*}}=A_{(0)}$ by $A_{0}^{k^{*}}, \ldots, A_{k^{*}}^{k^{*}}$. 
Now, $A_{(0)}=A_{k^{*}+1}^{k^{*}+1}<A_{k^{*}}^{k^{*}+1}$, so $A_{(0)}=A_{k^{*}}^{k^{*}}<A_{k^{*}}^{k^{*}+1}$. In fact, as is easily seen, this holds for all $j: A_{j}^{k^{*}}<A_{j}^{k^{*}+1}$. (Compare the optimal values in the case where jump $j$ occurs at (before) $t=0$ in the two problems where $\lambda_{m}=0, m \geq$ $k^{*}+1$, and where $\lambda_{m}=0, m \geq k^{*}+2$.) Assume now that $\lambda_{j}>0$ for all $j$ and let $A_{j}=\lim _{k^{*} \rightarrow \infty} A_{j}^{k^{*}}$. (It is shown below that, for some $A_{0}^{*}, A_{0}^{*} \geq A_{j}^{k^{*}}$, for all $k^{*}, j$.) Then, $\lim _{j \rightarrow \infty} A_{j}=A_{(0)}$, and the $A_{j}$ 's satisfy (14).

Let $x_{*}(t, \omega)$ be the solution for $u \equiv 0$. By (11), $x_{*}(t)=\sum_{i} x^{0} e^{k t}(1+\alpha)^{i} 1_{\left[\tau_{i}, \tau_{i+1}\right)}(t)=e^{k t} \Gamma$, where $\Gamma:=$ $(1+\alpha)^{i} 1_{\left[\tau_{i}, \tau_{i+1}\right)}(t)$. Choose the smallest $j^{*}$ such that $\alpha \lambda_{j^{*}}<$ $\delta-k \gamma$. If $j^{*}$ jumps occur at once at $0\left(\lambda_{j}=\infty, j \leq j^{*}\right)$, while further jumps occur with intensity $\lambda=\lambda_{j^{*}}$, then $E \Gamma$ would equal $(1+\alpha)^{j^{*}} \sum_{i=1}^{\infty}(1+\alpha)^{i}\left[(\lambda t)^{i} / i !\right] e^{-\lambda t}=(1+\alpha)^{j^{*}} e^{\alpha \lambda_{j^{*} t}}$. Hence, $E \Gamma^{\gamma} \leq(1+\alpha)^{j^{*}} e^{\alpha \lambda_{j^{*} t}}$. For any admissible solution $x(t, \omega), x(t, \omega) \leq x_{*}(t, \omega)$, for all $(t, \omega)$; hence, for $t=$ $T, 0 \leq E\left[e^{-\delta T} A_{j}(x(T, \omega))^{\gamma}\right] \leq E\left[e^{-\delta T} A_{0} e^{k \gamma T} \Gamma^{\gamma}\right] \leq$ $e^{-(\delta-k \gamma) T} A_{0}(1+\alpha)^{j^{*}} e^{\alpha \lambda_{j^{*} T}} \rightarrow 0$ when $T \rightarrow \infty$. So (A.7) in the appendix is satisfied. Hence, sufficient conditions hold (see Theorem 2 in Appendix) and $u_{j}=x\left(A_{j}\right)^{1 /(\gamma-1)}, j=$ $0,1, \ldots$, are optimal. That is, if $v^{*}(t, \omega)=\left(A_{j}\right)^{1 /(\gamma-1)} 1_{\left[\tau_{j}, \tau_{j+1}\right)}(t)$ and $x^{*}(t, \omega)=x_{0}(1+\alpha)^{i} e^{k t-\sum_{1 \leq j \leq i} A_{j-1}^{1 /(\gamma-1)}\left(\tau_{j}-\tau_{j-1}\right)-A_{i}^{1 /(\gamma-1)}\left(t-\tau_{i}\right)}$ for $t \in\left(\tau_{i}, \tau_{i+1}\right)$ (see (11)), then $u^{*}(t, \omega)=v^{*}(t, \omega) x^{*}(t, \omega)$ is optimal $\left(x^{*}(t, \omega)\right.$ evidently satisfies $x^{*}(t, \omega)>0$, for all $\left.t\right)$. Hence, $A_{j}=\widetilde{A}_{j}$. (In the appendix, for the sufficiency of the HJB equation to hold, it is required that $u^{*}(t, \omega)$ is bounded if $T<\infty$ (for $T=\infty$, we need boundedness for $t$ in all bounded intervals). This is not the case here. But we could have replaced $u$ by $v x, v$ being the control. Assume that we require $v(t, \omega)$ to be bounded in the above manner. Now, $v^{*}(t, \omega)$ is so bounded, and it is then optimal in the set of such bounded $v(\cdot, \cdot)$ 's.)

We can show that the $A_{j}$ 's are increasing in $\alpha$ and in each $\lambda_{i}, i>j$. The simplest argument is that this must be so, when we now know that $A_{j} y^{\gamma}$ is the optimal value function after $j$ jumps at (before) $t=0$.

If all $\lambda_{j}=\lambda<(\delta-k \gamma) / \alpha$, then, using (16), $x^{*}(t)=$ $x_{0}(1+\alpha)^{i} e^{k t-A_{(\lambda)}^{1 /(\gamma-1)} t}=x_{0}(1+\alpha)^{i} e^{-\rho^{*} t}$ when $t \in\left(\tau_{i}, \tau_{i+1}\right)$, where $0<\rho^{*}=\rho+\left[\lambda-\lambda(1+\alpha)^{\gamma}\right] /(1-\gamma)<\rho=(\delta-k) /(1-\gamma)$. (The inequality $\alpha \lambda<\delta-k \gamma$ implies $\delta-k \gamma+\lambda-\lambda(1+\alpha)^{\gamma}>\delta-$ $k \gamma+\lambda-\lambda(1+\alpha)>0$, so $A_{(\lambda)}>0$. In particular, $A_{\left(\lambda_{j^{*}}\right)}>0$. The above calculations show that in the problem where $\lambda_{j}=\lambda_{j^{*}}$, for $j>j^{*}$, the optimal value functions are $A_{j}^{*} y^{\gamma}$, where $A_{j}^{*}=A_{\left(\lambda_{j^{*}}\right)}$ for $j \geq j^{*}, A_{j}^{*}$ given by backward induction using (14) for $j<j^{*}$, again $A_{j-1}^{*} \geq A_{j}^{*}$. Evidently, $A_{j}^{*} \geq A_{j}$. Now, $A_{j} \leq A_{\left(\lambda_{j}\right)}$ for $j \geq j^{*}$ and, from (16), it follows that $A_{\left(\lambda_{j}\right)} \rightarrow A_{(0)}$ when $j \rightarrow \infty$.) Now, relate the present case to what happened in Model 1. As $u^{*}(t)=A_{(\lambda)}^{1 /(1-\gamma)} x^{*}(t)$ and $\rho^{*}<\rho$, in each interval $\left(\tau_{i}, \tau_{i+1}\right),(d / d t) u^{*}(t)>d / d t\left(b e^{-\rho t}\right)$ (be $e^{-\rho t}$ is the optimal control in Model 1); moreover, when $t$ passes each $\tau_{i} u^{*}(t)$ changes by a factor $(1+\alpha)$.

\section{Model 3}

In this model, the physical volume of oil production is constant $=1$, but the oil price $z$ jumps up and down at Poisson distributed time points $\tau_{i}, i=1,2, \ldots$. Let $\tau_{0}=0$. Consider the problem

$$
\begin{array}{r}
\max _{u(\cdot)} \int_{0}^{T} \gamma^{-1} e^{-\delta t} u^{\gamma} d t, \quad \gamma, \delta \text { given positive constants, } \\
\gamma \in(0,1), u \geq 0,
\end{array}
$$

where $u$ is the control, subject to the differential equations below and

$$
z\left(\tau_{i}+\right)=V_{i}
$$

where $V_{i}$ is taking a finite number of values $\left\{V^{j}\right\}_{j}$ with given probabilities $p_{j}$, all $V_{i}$ are identically distributed, and $\tau_{i}$ is exponentially distributed in $\left(\tau_{i-1}, \infty\right)$ with intensity $\lambda$ (all the random variables $\tau_{i}-\tau_{i-1}, V_{i}$ independent). Let $v=E V$. The two states $y$ and $z$ are governed by (18) (no jumps in $y$ ) and

$$
\dot{y}=a y+z-u, \quad \delta>a>0,
$$

$\dot{z}=0$, where $a$ is a given constant;

$y(0)=y_{0}, z(0)=z_{0}$, where $y_{0}$ and $z_{0}$ are given constants; in fact, it will be convenient to assume that $z_{0}=v$. The end constraint $E[y(T)] \geq 0$ is required to hold. Here, $y$ is wealth and $a y$ is interest earned.

Let us use the extremal method (see the appendix) for solving this problem. In what follows, it is guessed that adjoint functions do not depend on arbitrarily given initial points (which in the appendix are denoted by $(s, y)$ ). The adjoint function corresponding to $y$ is hence denoted by $p(t, i)$. We make the guess that $p(t, i)$ simply equals $K e^{-a t}$ (independent of $i), K$ being an unknown. It does satisfy the adjoint equation

$$
\dot{p}(t, i-1)=-a p(t, i-1)+\lambda p(t, i-1)-\lambda E p(t, i) .
$$

$\left(E p_{i}(t)\right.$, the expectation with respect to $V_{i}$, simply equals $p(t, i)$, since $p(t, i)$ does not depend on $V_{i}$.) The adjoint function $p^{z}(t, i)$ corresponding to the state variable $z$ satisfies $\dot{p}^{z}(t, i)=-p(t, i)+\lambda p^{z}(t, i)$, with $p^{z}(T, i)=0(z(T)$ is free $)$, we do not need the formula for $p^{z}(t, i)$.

Next, maximum of the Hamiltonian $H=e^{-\delta t} u^{\gamma} / \gamma+$ $p(a y+z-u)$ is obtained for $u$ satisfying the first order condition $u^{\gamma-1} e^{-\delta t}=p$, so $u=\left(e^{\delta t} p\right)^{1 /(\gamma-1)}=K^{\beta} e^{-\kappa t}$, where $\kappa=(a-\delta) /(\gamma-1)>0, \beta=1 /(\gamma-1)$.

Now, $\dot{y}=a y+z(t)-u=a y+z(t)-K^{\beta} e^{-\kappa t}$, so

$$
y(t)=C e^{a t}+e^{a t} \int_{0}^{t} z(s) e^{-a s} d s+\frac{K^{\beta} e^{-\kappa t}}{a+\kappa},
$$

where $C=y_{0}-K^{\beta} /(a+\kappa)$. 
Note that, for any $s, E z(s)=v$. We want $y(t)$ to satisfy the condition $E y(T)=0$; that is,

$$
\begin{aligned}
0 & =E\left\{y_{0} e^{a T}+e^{a T} \int_{0}^{T} z(s) e^{-a s} d s+\frac{K^{\beta} e^{-\kappa T}}{a+\kappa}-\frac{e^{a T} K^{\beta}}{a+\kappa}\right\} \\
& =e^{a T}\left\{y_{0}+\frac{v\left(1-e^{-a T}\right)}{a}+\frac{K^{\beta} e^{-(\kappa+a) T}}{a+\kappa}-\frac{K^{\beta}}{a+\kappa}\right\},
\end{aligned}
$$

which determines $K$. From now on, denote the solutions $y(t)$ and $z(t)$ by $y^{*}(t, \omega)$ and $z^{*}(t, \omega)$, with $u^{*}(t)=K^{\beta} e^{-\kappa t}$. Sufficient conditions (see Theorem 3 in the appendix) are easily seen to be satisfied. We need to check the end condition $E\left[p(T, \omega)\left(y(T, \omega)-y^{*}(T, \omega)\right)\right] \geq 0(p(\cdot, \omega)=p(\cdot))$ for all admissible solutions $y(t, \omega)$ (see (A.13) in the appendix). The terminal condition is $E y(T, \omega) \geq 0$, for any admissible $y(t, \omega)$, and by construction $y^{*}(t, \omega)$ satisfies $\operatorname{Ep}(T) y^{*}(T, \omega)=0$, so the just-mentioned end condition is satisfied. We hence have optimality of $u^{*}(t), y^{*}(t, \omega)$, and $z^{*}(t, \omega)$ among admissible triples $(u(t, \omega), y(t, \omega), z(t, \omega))$ for which $E y(T, \omega) \geq$ $0\left(z(t, \omega)=z^{*}(t, \omega)\right)$.

If $T=\infty$, the end condition required for using the sufficient condition in Theorem 2 in the appendix is $\liminf _{T \rightarrow \infty} E\left[p(T, \omega)\left(y(T, \omega)-y^{*}(T, \omega)\right)\right] \geq 0$. The condition $\lim _{T} E\left[p(T) y^{*}(T, \omega)\right]=0$ determines $K$; it now reduces to the condition $y_{0}+v / a=K^{\beta} /(a+\kappa)$. Moreover, we now have optimality among all triples $u(t, \omega), y(t, \omega), z(t, \omega)$ for which $\lim \inf _{T \rightarrow \infty} E[p(T) y(T, \omega)] \geq 0$. (This inequality means that the next-to-last inequality is satisfied.) Note that $K^{\beta}$ and so the control, quite expectably, are increasing in $v=E V$.

\section{Model 4}

In this example, $y$ is the rate of production (production per unit of time) of oil. The oil price $z$ jumps up or down between two values at Poisson distributed time points. The income the owner does not consume, that is, $z y-u$, can be used to increase the rate of production, with the change $\dot{y}$ being proportional to $z y-u$. (If $z y-u$ is negative, it means that he takes money out of his business and runs it down.) For simplicity, the proportionality factor is put equal to 1 , and, simply, $z$ jumps between the values 1 and 2 . So consider

$$
\max _{u(\cdot)} E\left[\int_{0}^{\infty} e^{-\delta t} u^{\gamma} d t\right],
$$

where $\delta$ and $\gamma$ are given positive constants, $\gamma \in(0,1)$,

$\delta>2 \gamma, u \geq 0$ the control, subject to

$$
\begin{gathered}
\dot{y}=z y-u, \quad y(t) \geq 0 \text { a.s., } \\
\dot{z}=0, \quad z(0)=1, \quad z(T) \text { free, } \\
z\left(\tau_{i}+\right)=V_{i}, \quad V_{i} \in\{1,2\}, \quad \operatorname{Pr}\left[V_{i}=1\right]=\frac{1}{2},
\end{gathered}
$$

where $\tau_{i}$ is exponentially distributed in $\left(\tau_{i-1}, \infty\right)$ with intensity $\lambda$ (all $V_{i}, \tau_{i}-\tau_{i-1}$ independent $V_{0}=1, z_{0}=0$ ). There are no jumps in $y$ and $y(0)=y_{0}>0, y_{0}$ being fixed.

Let $J(y, j), j=1,2$, be the currentoptimal value function sought, where $j=z \in\{1,2\}$. The current value HJB equation is as follows

$$
\begin{aligned}
\delta J(y, j)= & \max \left\{u^{\gamma}+J_{y}(y, j)(j y-u)\right\} \\
& +\left(\frac{1}{2}\right) \lambda J(y, 1)+\left(\frac{1}{2}\right) \lambda J(y, 2)-\lambda J(y, j) .
\end{aligned}
$$

Let us $\operatorname{try} J(y, 1)=A_{1} y^{\gamma}, J(y, 2)=A_{2} y^{\gamma}$. Then, the maximizing $u$ equals $u=B_{j} y, B_{j}=\left(A_{j}\right)^{1 /(\gamma-1)}$. In the two cases $j=1$ and $j=2$, we get the two equations

$$
\begin{aligned}
0= & -\delta A_{1}+A_{1}^{\gamma /(\gamma-1)}+\gamma A_{1} \\
& -\gamma A_{1}\left(A_{1}\right)^{1 /(\gamma-1)}+\left(\frac{A_{1}}{2}+\frac{A_{2}}{2}\right) \lambda-\lambda A_{1}, \\
0= & -\delta A_{2}+A_{2}^{\gamma /(\gamma-1)}+2 \gamma A_{2} \\
& -\gamma A_{2}\left(A_{2}\right)^{1 /(\gamma-1)}+\left(\frac{A_{1}}{2}+\frac{A_{2}}{2}\right) \lambda-\lambda A_{2} .
\end{aligned}
$$

The equations can be solved for positive $A_{1}$ and $A_{2}$ (shown in a moment). Automatically, the solution $y^{*}(t, \omega)$ corresponding to $u(y, z)=u(y, j)=B_{j} y$ satisfies $y^{*}(t, \omega) \geq$ 0 a.s., for all $t$.

To show the existence of a solution of (26) and (27), note that if we put $A_{2}=A_{1}$ in (26), then by dividing by $A_{1}$, we get $0=-\delta+\gamma+(1-\gamma) A_{1}^{1 /(\gamma-1)}=: \kappa_{1}\left(A_{1}\right)$, and $\kappa_{1}\left(A_{1}\right)=0$ yields $A_{1}=A_{1}^{*}:=[(\delta-\gamma) /(1-\gamma)]^{\gamma-1}$. Similarly, if we put $A_{1}=A_{2}$ in (27), we get $0=-\delta+2 \gamma+(1-$ $\gamma) A_{2}^{1 /(\gamma-1)}=: \kappa_{2}\left(A_{2}\right)$, and $\kappa_{2}\left(A_{2}\right)=0$ yields $A_{2}=A_{2}^{*}:=$ $[(\delta-2 \gamma) /(1-\gamma)]^{\gamma-1}$. Now, $A_{1}^{*} / A_{2}^{*}=[(\delta-2 \gamma) /(\delta-\gamma)]^{1-\gamma}<$ 1. Denote the right-hand sides in (26) and (27) by $\phi\left(A_{1}, A_{2}\right)$ and $\psi\left(A_{2}, A_{1}\right)$, respectively. Both are increasing in the second place and decreasing in the first place. When $A_{1} \in\left[A_{1}^{*}, A_{2}^{*}\right]$, $\psi\left(A_{2}^{*}, A_{1}\right) \leq \psi\left(A_{2}^{*}, A_{2}^{*}\right)=0$, and $\psi\left(A_{1}^{*}, A_{1}\right) \geq \psi\left(A_{1}^{*}, A_{1}^{*}\right)>$ $\phi\left(A_{1}^{*}, A_{1}^{*}\right)=0$ (the only difference in $\psi(A, A)$ and $\phi(A, A)$ is the third term in the formulas). Thus, $\psi\left(A_{2}, A_{1}\right)=0$ can be uniquely solved with respect to $A_{2}$ for $A_{1}$ in $\left[A_{1}^{*}, A_{2}^{*}\right]$, denote the solution $A_{2}\left(A_{1}\right)$, it is evidently increasing in $A_{1}$ and $A_{2}\left(A_{1}\right) \in\left[A_{1}^{*}, A_{2}^{*}\right], A_{2}\left(A_{2}^{*}\right)=A_{2}^{*}$. Moreover, $A_{1}<$ $A_{2}^{*}, \Rightarrow 0<\kappa_{2}\left(A_{1}\right)=\psi\left(A_{1}, A_{1}\right) \Rightarrow A_{2}\left(A_{1}\right)>A_{1}$. Consider the function $\beta\left(A_{1}\right):=\phi\left(A_{1}, A_{2}\left(A_{1}\right)\right)>\phi\left(A_{1}, A_{1}\right)$. Now, $\beta\left(A_{1}^{*}\right)>\phi\left(A_{1}^{*}, A_{1}^{*}\right)=0$ and $\beta\left(A_{2}^{*}\right)=\phi\left(A_{2}^{*}, A_{2}^{*}\right)<$ $\psi\left(A_{2}^{*}, A_{2}^{*}\right)=0$, so $A_{1} \in\left(A_{1}^{*}, A_{2}^{*}\right)$ exists for which $0=$ $\beta\left(A_{1}\right)=\phi\left(A_{1}, A_{2}\left(A_{1}\right)\right)$. So this $A_{1} \in\left(A_{1}^{*}, A_{2}^{*}\right)$ and $A_{2}=$ $A_{2}\left(A_{1}\right) \in\left(A_{1}^{*}, A_{2}^{*}\right), A_{2}>A_{1}$, satisfy both (27) and (26).

For any admissible $y(t, \omega), 0 \leq E\left[e^{-\delta T} A_{j}(y(T, \omega))^{\gamma}\right] \leq$ $A_{2}^{*} e^{-\delta T}\left(y_{*}(T)\right)^{\gamma}$, where $y_{*}(t)$ is the solution of $\dot{y}=2 y$ (i.e., $\left.y_{*}(T)=y_{0} e^{2 t}\right)$, so, for all admissible $y(t, \omega), 0 \leq$ 
$\lim _{T \rightarrow \infty} E\left[e^{-\delta T} A_{j}(y(T, \omega))^{\gamma}\right] \leq \lim _{T \rightarrow \infty} e^{-\delta T} A_{2}^{*}\left(y_{*}(T)\right)^{\gamma}=$ $\lim _{T \rightarrow \infty} A_{2}^{*} y_{0}^{\gamma} e^{(2 \gamma-\delta) T}=0$. Hence, (A.7) in the appendix holds; the sufficient conditions in Theorem 2 are satisfied, and if $u=y\left(A_{j}\right)^{1 /(\gamma-1)}$ when $V_{i}=j \in\{1,2\}$, then $u$ is optimal. (Hence, if $v(t, \omega):=\sum_{i=0}^{\infty}\left(A_{1}^{1 /(\gamma-1)} 1_{\{V=1\}}\left(V_{i}\right)+\right.$ $\left.A_{2}^{1 /(\gamma-1)} 1_{\{V=2\}}\left(V_{i}\right)\right) 1_{\left[\tau_{i}, \tau_{i+1}\right)}(t)$ and $y^{*}(t, \omega)$ is the solution of $\dot{y}=$ $z(t, \omega) y-v(t, \omega) y$, then $u^{*}(t, \omega)=v(t, \omega) y^{*}(t, \omega)$ is optimal. $)$

Define $A^{*}=[(\delta-3 \gamma / 2) /(1-\gamma)]^{\gamma-1}$ (i.e., $A^{*}$ satisfies $\left.0=-\delta A+A^{\gamma /(\gamma-1)}+3 \gamma A / 2-\gamma A A^{1 /(\gamma-1)}\right)$. When $\lambda=0$ then $A_{i}=A_{i}^{*}$, and one may show that when $\lambda>0$ decreases, $A_{2}$ increases towards $A_{2}^{*}$ while $A_{1}$ decreases towards $A_{1}^{*}$. When $\lambda$ increases to infinity, $A_{1}$ increases to $A^{*}$, and $A_{2}$ decreases to $A^{*}$. In fact, $A_{1} \in\left(A_{1}^{*}, A^{*}\right)$ and $A_{2} \in\left(A^{*}, A_{2}^{*}\right)$. To prove convergence to $A^{*}$, note first that $A_{1}, A_{2}$ are bounded uniformly in $\lambda$. If $A_{1}$ and $A_{2}$ did not converge to a common number, say $A_{*}$, when $\lambda \rightarrow \infty$, the two last terms in (27) and (26) would blow up to infinity for (certain) large values of $\lambda$, while the remaining terms would be bounded, a contradiction. Summing the two equations in (27) and (26) gives $0=-\delta A_{1}+A_{1}^{\gamma /(\gamma-1)}+\gamma A_{1}-\gamma A_{1} A_{1}^{1 /(\gamma-1)}-\delta A_{2}+$ $A_{2}^{\gamma /(\gamma-1)}+2 \gamma A_{2}-\gamma A_{2} A_{2}^{1 /(\gamma-1)}$. Hence, $A_{*}$ satisfies $0=-2 \delta A_{*}+$ $2 A_{*}^{\gamma /(\gamma-1)}+3 \gamma A_{*}-2 \gamma A_{*} A_{*}^{1 /(\gamma-1)}$; that is, $A_{*}=A^{*}$.

Two very simple models discussed below contain the feature that the owner can influence the chance of discovery but that it is costly to do so. In the first one, the intensity of discoveries $\lambda$ is influenced by how much money is put into search at any moment in time; in the second one, it is costly buildup of expertise that matters for the intensity of discoveries.

\section{Model 5}

Consider the problem

$$
\max _{u(\cdot)} E\left[\sum_{i=1}^{\infty} e^{-\delta \tau_{i}} g_{0}-\int_{0}^{\infty} e^{-\delta t} u d t\right],
$$

where $\delta$ is a fixed positive number,

$$
x\left(\tau_{i}+\right)-x\left(\tau_{i}^{-}\right)=\alpha
$$

where $\alpha$ is a fixed positive number, $x(0)=0, \dot{x}=0$,

and $\lambda=\mu u^{1-\kappa} /(1-\kappa)$, where $\mu>0, \kappa$ are given constants, $\kappa \in(0,1), u \geq 0$ is the control, and $\lambda$ is the intensity of jumps. All fields found are of the same size $\alpha$. We imagine that fields are sold immediately when they are found or that they are produced over a fixed period of time, with a fixed production profile. In both cases, we let income from a field discounted back to the time of discovery be equal to $g_{0}:=\beta \alpha$, for a given $\beta>0$. The oil owner wants to maximize the sum of discounted incomes earned over $[0, \infty)$.

Let us try the proposal that the current value optimal value function $J=a$, where $a$, an unknown. The current value
HJB equation is

$$
\delta a=\max _{u}\left\{-u+\frac{\mu g_{0} u^{1-\kappa}}{1-\kappa}\right\},
$$

which implies that the maximizing $u$ satisfies $\mu g_{0} u^{-\kappa}=1$, so $u=\left(\mu g_{0}\right)^{1 / \kappa},-u+\mu g_{0} u^{1-\kappa} /(1-\kappa)=-\left(\mu g_{0}\right)^{1 / \kappa}+$ $\mu g_{0}\left(\left(\mu g_{0}\right)^{1 / \kappa}\right)^{1-\kappa} /(1-\kappa)=(\kappa /(1-\kappa))\left(\mu g_{0}\right)^{1 / \kappa}=: \widehat{a}$, and $a=$ $\widehat{a} / \delta$. Trivially, (A.7) in the appendix holds, so the sufficient conditions in Theorem 2 are satisfied and $u=\left(\mu g_{0}\right)^{1 / \kappa}$ is optimal. Because $a>0$, in expectation (but not in all runs), over $[0, \infty)$, a positive sum of discounted incomes $(=a)$ has been earned.

Often, it is the case that the best fields are exploited first hence, $x\left(\tau_{i}+\right)-x\left(\tau_{i}-\right)=\alpha_{i}$, where $\alpha_{i}$ is decreasing and even $\alpha_{i} \downarrow 0$. Then, when $j$ jumps are imagined to have happened already at time 0 , we guess $J(j)=a_{j}$. Define $g_{0}^{i}=\beta \alpha_{i}$. Then, the HJB equation isvspace-11pt

$$
\begin{aligned}
\delta a_{j} & =\max _{u}\left\{-u+\frac{\mu\left(g_{0}^{j+1}+a_{j+1}-a_{j}\right) u^{1-\kappa}}{1-\kappa}\right\} \\
& =\left(\frac{\kappa}{1-\kappa}\right)\left(\mu g_{0}^{j+1}+a_{j+1}-a_{j}\right)^{1 / \kappa}=: v_{j}\left(a_{j}\right),
\end{aligned}
$$

and $u_{j}=\left(\mu g_{0}^{j+1}+a_{j+1}-a_{j}\right)^{1 / \kappa}$ yields maximum. Postulate for the moment that, for some $j, a_{j+1} \in\left[a_{j+2}, g_{0}^{j+2}+a_{j+2}\right)$. Then, $\delta a_{j+1}=v_{j+1}\left(a_{j+1}\right) \leq(\kappa /(1-\kappa))\left(\mu g_{0}^{j+2}\right)^{1 / \kappa}<(\kappa /(1-$ $\kappa))\left(\mu g_{0}^{j+1}\right)^{1 / \kappa}=v_{j}\left(a_{j+1}\right)$. Thus, if we try $a_{j}=a_{j+1}$, we get $\delta a_{j}<v_{j}\left(a_{j}\right)$. If we try $a_{j}=g_{0}^{j+1}+a_{j+1}$, we get $\delta a_{j}>v_{j}\left(a_{j}\right)=0$. So a correct value of $a_{j} \in\left(a_{j+1}, g_{0}^{j+1}+a_{j+1}\right)$ exists such that $\delta a_{j}=v\left(a_{j}\right)$. This continues backwards and gives $a_{0}>a_{1}>$ $\cdots>a_{j+1}$. If, for some $j^{*}, \alpha_{j}=0$, for $j \geq j^{*}+1$, then $a_{j^{*}}=$ $a_{j^{*}+1}=a_{j^{*}+2}=\cdots=0$, so in this case, (30) gives $0<a_{j^{*}-1}<$ $a_{j^{*}-2}<\cdots<a_{0}$. If all $\alpha_{j}>0$, letting $j^{*} \rightarrow \infty$, it is easily seen that there exists an infinite decreasing sequence $a_{j} \downarrow 0$ when $j \rightarrow \infty$ satisfying (30) (compare a similar argument in Model 2).

Still, trivially condition (A.7) below holds, sufficient conditions in Theorem 2 in the appendix are satisfied and $u_{j}=\left(\mu g_{0}^{j+1}+a_{j+1}-a_{j}\right)^{1 / \kappa}$ on $\left[\tau_{j}, \tau_{j+1}\right), j=0,1,2, \ldots$, are optimal. (Uniqueness of the $a_{j}$ 's follows from optimality.)

\section{Model 6}

Consider the problem

$$
\max _{u(\cdot)} E\left[\sum_{i=1}^{\infty} e^{-\delta \tau_{i}} g_{0}-\int_{0}^{\infty} e^{-\delta t} u d t\right]
$$

where $\delta$ is a fixed positive number,

$$
x\left(\tau_{i}+\right)-x\left(\tau_{i}-\right)=\alpha
$$


where $\alpha$ is a fixed positive number, $x(0)=0, \dot{x}=0$,

$\dot{y}=\frac{u^{1-\kappa}}{1-\kappa}, \quad$ where $u \geq 0, u$ is the control, $y(0)=0$,

no jumps in $y, \lambda=\mu y, \mu, \kappa$ are fixed positive numbers, $\kappa<1$, $\lambda$ is the intensity of jumps in $x$.

All fields found are of the same size $\alpha$. We again imagine that fields are sold immediately when they are found or that they are produced over a fixed period of time, with a fixed production profile. Let income from a field discounted back to the time of discovery be equal to $g_{0}:=\beta \alpha$, for a given $\beta>0$. The state $y(t)$ is the amount of expertise available for finding new fields, built up over time according to $\dot{y}=$ $u^{1-\kappa} /(1-\kappa)$, where $u$ is money per unit of time spent on building expertise.

Let us try the proposal that the current value function $J$ equals $a+k y, a$ and $k$ being unknowns. The current value HJB equation is

$$
\delta(a+k y)=\max _{u}\left\{-u+\frac{k u^{1-\kappa}}{1-\kappa}+\mu y g_{0}\right\} .
$$

Then, $k \delta=\mu g_{0}$, so $k=\mu g_{0} / \delta$. Moreover, the maximizing $u$ equals $u=k^{1 / \kappa}$, and $-u+k u^{1-\kappa} /(1-\kappa)=(\kappa /(1-\kappa)) k^{1 / \kappa}=: \check{a}$, $a=\check{a} / \delta$. The optimal solution $y^{*}(t)$ equals $t b$, where $b=$ $k^{(1-\kappa) / \kappa} /(1-\kappa)$. Evidently, (A.7) below is satisfied, because $J=$ $e^{-\delta T}(a+k y(T)) \geq 0$ for any admissible $x(\cdot), y(\cdot)$ and $e^{-\delta T}(a+$ $\left.k y^{*}(T)\right) \rightarrow 0$ when $T \rightarrow \infty$. Hence, $u=k^{1 / \kappa}=\left(\mu g_{0} / \delta\right)^{1 / \kappa}$ is optimal.

\section{Comparisons}

In Models 1, 2, 5, and 6, oil finds are made at stochastic points in time; in Models 3 and 4, it is the price of oil that changes at stochastic points in time. In Model 1 , we operate with the constraint $\operatorname{Ex}(\infty, \omega) \geq 0$ (for $T=\infty, a=0$ ), where $x$ is the oil-owners' wealth. Here, for some runs, $x(\infty, \omega)$ can be negative $\left(\beta>x_{0}\right)$ and, for other runs, positive. In Model 2, we required $x(t, \omega)>0$ for all $t$, all $\omega$. (The results in that model would have been the same if we had required only $x(\infty, \omega) \geq 0$ for all $\omega$.) In Model 2, the optimal control comes out as stochastic and not deterministic as in Model 1. Moreover, as a comment in Model 2 says, as a function of time, the optimal control decreases more rapidly in Model 1 as compared to Model 2. The latter feature stems from the fact that, in Model 2, we enhance future income prospects by not decreasing $x$ too fast, because the jump term (the right-hand side of the jump equation) depends positively on $x$, which is not the case in Model 1.

In Models 3 and 4, the oil price exhibits sudden stochastic jumps. In Model 3, the rate of oil production is constant, but income earned (as well as interest) is placed in a bank after subtraction of consumption. In Model 4, income earned, after subtraction of consumption, is reinvested in the oil firm to increase production. In Model 4, the optimal control is stochastic; it depends on whether the current price is high or low. In Model 3, the control is deterministic, and it depends only on the expectation $v$ of the stochastic price. Consider the case where, in Model 3, the expected price $v$ is zero and $a=3 / 2$. Then, $u=K^{\beta} e^{-\kappa t}$, where $\kappa=(3 / 2-\delta) /(\gamma-1)$ and $K^{\beta}=y_{0}(3 / 2+\kappa)$. We saw in Model 4 that when the intensity of jumps $\lambda$ is very high, $A_{1} \simeq A_{2} \simeq A^{*}$, where $\left.A^{*}=[\delta-3 \gamma / 2) /(1-\gamma)\right]^{\gamma-1}$; we hardly pay attention to what the current price is, because it changes so frequently. Now $u=y\left(A_{j}\right)^{1 /(\gamma-1)}$ when the current price is $j(=z)$, or $u \simeq y\left(A^{*}\right)^{1 /(\gamma-1)}$ when $\lambda$ is large. When $\lambda$ is large (so $z$ switches very frequently between 1 and 2$)$, the stochastic path of the equation $\dot{y}=z y-u=z y-y\left(A^{*}\right)^{1 /(\gamma-1)}$ most often is very close to the deterministic path of $\dot{y}=(E z) y-u=$ $(3 / 2) y-y\left(A^{*}\right)^{1 /(\gamma-1)}=(3 / 2) y-[(\delta-3 \gamma / 2) /(1-\gamma)] y=-\kappa y$. The latter equation has the solution $y=y_{0} e^{-\kappa t}$, and the corresponding $u$ equals $(3 / 2) y-\dot{y}=(3 / 2) y_{0} e^{-\kappa t}+\kappa y_{0} e^{-\kappa t}=$ $\kappa y_{0} e^{-\kappa t}=y_{0}(3 / 2+\kappa) e^{-\kappa t}$, the same control as that obtained in Model 3 in the case $a=3 / 2, v=0$.

In the extremely simple Models 5 and 6, the frequency of oil finds is not fixed but influenced by a control. In Model 5 , the current frequency (or intensity) is determined by how much money is put into search at that moment in time. In the simplest case considered in Model 5, a find today does not influence the possibility of making equally sized discoveries tomorrow. Then it, is not unreasonable that the optimal control (which equals $\left(\mu g_{0}\right)^{1 / \kappa}$ ) is independent of the discount rate $\delta$ but dependent on the fixed value of the finds $g_{0}=\beta \alpha$. (Here, actually $\alpha$ could be the expected size of a find, in both Model 5 and Model 6; we could have had the sizes of the finds being independently stochastic, with $\alpha$ being the expected value of the sizes.) In Model 6, it is the ability to discover oil that is built up over time, so with greater impatience (higher $\delta$ ), we should expect less willingness of devoting money to increase this ability, and this shows up in the formula $u=\left(\mu g_{0} / \delta\right)^{1 / \kappa}$.

\section{Appendix}

Consider the problem

$$
\dot{x}=f(t, x, u), \quad t \in[0, T], x(0)=x^{0} \in \mathbb{R}^{n}, u \in U \subset \mathbb{R}^{k},
$$

$$
x\left(\tau_{j}+\right)-x\left(\tau_{j}-\right)=g\left(\tau_{j}, x\left(\tau_{j}^{-}\right), V_{j}\right), \quad V_{j} \in \mathbb{R}^{k^{*}}
$$

$x^{0}, U, T>0$ fixed, $u$ is the control, with criterion

$$
\begin{gathered}
E\left[\int_{[0, T]} f_{0}(t, x(t), u(t)) d t+h_{0}(x(T-))\right. \\
\left.\quad+\sum_{\tau_{j}<T} g_{0}\left(\tau_{j}, x\left(\tau_{j}-\right), V_{j}\right)\right] .
\end{gathered}
$$

Here, $\tau_{j}$ and $V_{j}, j=1,2, \ldots$, are random variables, with all $\tau_{j}-\tau_{j-1}$ and $V_{j}$ being independent $\left(\tau_{0}=0\right)$. Given $\tau_{j-1}$, 
the intensity of the jump $\tau_{j}$ in $\left[\tau_{j-1}, \infty\right)$ is $\lambda_{j}(t, x, u)$. (So for given nonanticipative function $x(t)=x(t, \omega), u(t)=$ $u(t, \omega)$, for $\tau \geq \tau_{j-1}$, the probability distribution of $\tau=\tau_{j}$ is $\left.\lambda_{j}(\tau, x(\tau), u(\tau)) e^{-\int_{\tau_{j-1}}^{\tau} \lambda_{j}(s, x(s), u(s)) d s}.\right)$

Assume that the five given functions $f_{0}, f, g_{0}, g$, and $h_{0}$ are continuous and Lipschitz continuous with respect to $x$ with a common rank $\kappa^{n}$ for $u \in U \cap B(0, n)$, independently of $(t, u, V)$, and also that these functions satisfy an inequality of the form $|\phi(t, x, u, V)| \leq \alpha_{n}+\kappa_{n}|x|$ for all $(t, x, u)$, $u \in U \cap B(0, n)$, all $V$. Also, $\lambda_{j}(\cdot, \cdot, \cdot)$ is continuous and $\sup _{t, j, x, u \in B\left(0, n^{\prime}\right)} \lambda_{j}(t, x, u)<\infty$ for all $n^{\prime}$. Define $u(t, \omega)$ to be admissible if $u(t, \omega) \in U$, for all $(t, \omega), \sup _{t, \omega}|u(t, \omega)|<\infty$, and if $u(t, \omega)$ is nonanticipative and is separately piecewise continuous in each real variable on which it depends. Then, corresponding to $u(t, \omega)$, for each $\omega$, there exists a nonanticipative function $x(t, \omega)$ (also called admissible), which is piecewise continuous in $t$, satisfying (A.2) and (A.1), for $t \in\left(\tau_{j}, \tau_{j+1}\right), j=0,1, \ldots(x(t, \omega)$ becomes piecewise continuous in each real variable on which it depends). If there are terminal conditions in the problem, $u(t, \omega)$ and $x(t, \omega)$ are called admissible if also the terminal conditions are satisfied. For pairs $u(t, \omega)$ and $x(t, \omega)$ to be called admissible, we can allow additional restrictions; namely, for given sets $Q_{j}(t) \subset$ $\mathbb{R}^{n}, t \in(0, T), j=0,1, \ldots$, it can be required that $x(t, \omega)$ has to belong to $Q_{j}(t)$ a.s. for $t \in\left(\tau_{j}, \tau_{j+1}\right)$.

HJB Equations. The following condition is sufficient for optimality. (Below, $j=0,1,2, \ldots$ indicates the number of jumps that have already happened).

Theorem 1. Assume that there exist functions $J(s, y, j)$ and controls $u_{j}(t, x), j=0,1,2, \ldots$, with $J(T, y, j)=h_{0}(y), C^{1}$ in $(s, y) \in[0, T] \times R^{n}, j=0,1,2, \ldots$, such that the functions $J(s, y, j)$, for all $(s, y)$, satisfy the HJB equation

$$
\begin{aligned}
0=J_{s}(s, y, j) & \\
+\max _{u \in U} & \left\{f_{0}(s, y, u)+J_{y}(s, y, j) f(s, y, u)\right. \\
& -\lambda_{j+1}(s, y, u) J(s, y, j) \\
& +\lambda_{j+1}(s, y, u) E\left[g_{0}\left(s, y, V_{j+1}\right)\right. \\
& \left.\left.+J\left(s, y+g\left(s, y, V_{j+1}\right), j+1\right)\right]\right\},
\end{aligned}
$$

and assume that each $u_{j}(s, y)$ yields maximum in this equation. Moreover, assume that a solution $x^{*}(t, \omega)$ of $\dot{x}=$ $\sum_{j=0}^{\infty} f\left(t, x, u_{j}(t, x)\right) 1_{\left(\tau_{j}, \tau_{j+1}\right]}(t)\left(\tau_{0}=0\right)$, with jumps given by (A.2), exists such that $\sup _{j \geq 0, t, \omega}\left|u_{j}\left(t, x^{*}(t, \omega)\right) 1_{\left(\tau_{j}, \tau_{j+1}\right]}(t)\right|<$ $\infty$. Assume also that, for some constants $a_{J}$ and $b_{J}$, $|J(s, y, j)| \leq a_{J}+b_{J}|y|$ for all $(s, y, j)$. Then, $u^{*}(t, \omega):=$ $\sum_{j=0}^{\infty} u_{j}\left(t, x^{*}(t, \omega)\right) 1_{\left(\tau_{j}, \tau_{j+1}\right]}(t)$ is optimal.

See pages 147 and 155 (and for a proof, see page 168) in [6]. (Formally, we only need to assume that the nonanticipative function $u^{*}(t, \omega)$ is measurable and bounded.)

In case of restrictions of the form $x(t, \omega) \in Q_{j}(t)$, we must assume that $x^{*}(t, \omega)$ satisfies these restrictions, in this case $J(s, y, j)$ needs to only be $C^{1}$ in a neighborhood of each point in $Q_{j}^{*}=\left\{(s, y): s \in(0, T), y \in Q_{j}(s)\right\}$, with $J(s, y, j) C^{0}$ in $\mathrm{cl}_{j}^{*}(\mathrm{cl}=$ closure $)$. Then, (A.4) needs to hold only at each point in $Q_{j}^{*}$.

If there are terminal conditions in the problem, replace $J(T, x, j)=h_{0}(x)$ by

$$
\begin{aligned}
& E\left[\sum_{j=0}^{\infty}\left\{J(T, x(T, \omega), j)-J\left(T, x^{*}(T, \omega), j\right)\right\} 1_{\left(t_{j}, \tau_{j+1}\right]}(T)\right] \\
& \quad \geq 0
\end{aligned}
$$

for all admissible $x(\cdot, \cdot)$.

If $h_{0} \equiv 0,[0, T]$ is replaced by $[0, \infty)$ and if $f_{0}=$ $e^{-\beta t} f^{0}(x, u), g_{0}=e^{-\beta t} g^{0}(x, V), \beta>0$, and $g, \lambda_{j}$, and $f$ are independent of $t$, and given a sequence $u_{j}(x)$, then a sufficient condition for optimality of $u^{*}(t, \omega)$ (catching up optimality in case infinite values of the criterion appear) is as follows.

Theorem 2. For $j=0,1,2, \ldots$, assume that some functions $u_{j}(y)$ and $\widetilde{J}(y, j)\left(C^{1}\right.$ in $\left.y\right)$ exist, such that $\widetilde{J}(y, j)$ satisfy the current value $H J B$ equation

$$
\begin{aligned}
& 0=-\beta \widetilde{J}(y, j) \\
& +\max _{u}\left\{f^{0}(y, u)+\widetilde{J}_{y}(y, j) f(y, u)\right. \\
& +\lambda_{j+1}(y, u) E\left[g^{0}\left(y, V_{j+1}\right)\right]+\lambda_{j+1}(y, u) \\
& \quad \times E\left[\widetilde{J}\left(y+g\left(y, V_{j+1}\right), j+1\right)\right. \\
& -\widetilde{J}(y, j)]\},
\end{aligned}
$$

for $j=0,1,2, \ldots$, with $u_{j}(y)$ yielding maximum. The functions $g^{0}, f^{0}, f, g$, and $\widetilde{J}$ are postulated to be continuous and Lipschitz continuous in $x$ with rank $\kappa^{n}$ for $u \in U \cap B(0, n)$ and all $V$ and to satisfy a growth conditions of the form $|\phi(x, u, V)| \leq \alpha_{n}+\kappa_{n}|x|$ for all $V, x, u \in U \cap B(0, n)$. Finally, $\sup _{j, x, u \in B\left(0, n^{\prime}\right)} \lambda_{j}(x, u)<\infty$ for each $n^{\prime}$. (It suffices to assume that, for any admissible pair $(x(t, \omega), u(t, \omega)$, for $t$ in bounded intervals, $\sup _{t, \omega} \lambda_{j}(x(t, \omega), u(t, \omega))<\infty$.) Assume that a solution $x^{*}(t, \omega)$ of $\dot{x}=\sum_{j} f\left(x, u_{j}(x)\right) 1_{\left(\tau_{j}, \tau_{j}+1\right]}(t)\left(\tau_{0}=\right.$ 0 ), with jumps given by (A.2), exists. Assume also that, for some constants $a_{J}$ and $b_{J},|\widetilde{J}(y, j)| \leq a_{J}+b_{J}|y|$ for all $(y, j)$. Define $u^{*}(t, \omega):=\sum_{j}^{\infty} u_{j}\left(x^{*}(t, \omega)\right) 1_{\left(\tau_{j}, \tau_{j}+1\right]}(t)$ and assume that $\sup _{t, \omega}\left|u^{*}(t, \omega)\right|<\infty$ for $t$ in bounded intervals. (If restrictions $x(t, \omega) \in Q_{j}, t \in\left(\tau_{j}, \tau_{j+1}\right)$ are present, one must have $x^{*}(t, \omega) \in Q_{j}$; in this case, it suffices that $\widetilde{J}(y, j)$ is $C^{1}$ in 
some open set containing $Q_{j}$, with $\widetilde{J}(y, j) C^{0}$ in $\mathrm{cl}_{j}$, and (A.6) needs to hold only in $Q_{j}$.) Then, $u^{*}(t, \omega)$ is optimal, provided

$$
\begin{aligned}
\liminf _{T \rightarrow \infty} E\left[e^{-\beta T} \sum_{j=0}^{\infty}\{\tilde{J}(x(T, \omega), j)\right. \\
\left.\left.\quad-\widetilde{J}\left(x^{*}(T, \omega), j\right)\right\} 1_{\left(t_{j}, \tau_{j+1}\right]}(T)\right] \geq 0,
\end{aligned}
$$

for all admissible $x(\cdot, \cdot)$.

(This is the infinite horizon current value form of (A.4); compare (3.71) and (3.69), page 150 in [6].)

The Extremal Method. The so-called extremal method (see page 117 and pages 126-130 in [6]) now to be described yields solutions satisfying a maximum principle (necessary condition). Now, it is assumed that $\sup _{j} \lambda_{j}<\infty$ and that $\lambda_{j}$ does not depend on $(t, x, u)$. To the assumptions above on $f_{0}, f, g_{0}, g$, and $h_{0}$, we now add the assumption that these five functions have derivatives with respect to $x$ that are continuous in $(t, x, u, V)$. Let $I$ be the identity matrix and let $H=f_{0}+p f$. For arbitrary $(s, y)$, we seek controls $u(t ; s, y, j)$ and solutions $x(t ; s, y, j), j=0,1, \ldots, u(t ; s, y, j)$ yielding maximum of $H(t, x(t ; s, y, j), u, p(t ; s, y, j))$, where each $p(t)=p(t ; s, y, j)$ is a solution on $[s, T]$ of

$$
\begin{aligned}
\dot{p}=- & H_{x}(t, x(t ; s, y, j), u(t ; s, y, j), p)+\lambda_{j+1} p \\
-\lambda_{j+1} E[ & \left.g_{0 x}\left(t, x(t ; s, y, j), V_{j+1}\right)\right] \\
-\lambda_{j+1} E[ & {\left[t\left(t ;, x+g\left(t, x, V_{j+1}\right), j+1\right)\right.} \\
& \left.\times\left(I+g_{x}\left(t, x, V_{j+1}\right)\right)\right]_{x=x(t ; s, y, j)}
\end{aligned}
$$

with end condition $p(T)=\left[h_{0 x}(x)\right]_{x=x(T ; s, y, j)}$ and where each $x(t)=x(t ; s, y, j)$ is a solution of $\dot{x}=f\left(t, x, u_{j}(t ; s, y, j)\right)$ with initial condition $x(s ; s, y, j)=y(j=$ the number of jumps that has happened).

Frequently, to find $x(t ; s, y, j), u(t ; s, y, j)$, and $p(t ; s, y, j)$, as in deterministic problems, one first seeks a control $\widehat{u}(t, x, p)$ that maximizes $H(t, x, u, p)$, and then one solves the equations $\dot{x}=f(t, x, \widehat{u}(t, x, p))$ and

$$
\begin{aligned}
\dot{p}=-H_{x}(t, x, \widehat{u} & (t, x, p), p)+\lambda_{j+1} p \\
-\lambda_{j+1} E[ & g_{0 x}\left(t, x, V_{j+1}\right) \\
& +p\left(t ; t, x+g\left(t, x, V_{j+1}\right), j+1\right) \\
& \left.\times\left(I+g_{x}\left(t, x, V_{j+1}\right)\right)\right],
\end{aligned}
$$

with side conditions

$$
x(s ; s, y, j)=y, \quad p(T)=\left[h_{0 x}(x)\right]_{x=x(T)} .
$$

Evidently, to solve the equations $\dot{x}=f(t, x, \widehat{u}(t, x, p))$ and (A.9) to obtain $x(t)=x(t ; s, y, j)$ and $p(t)=p(t ; s, y, j)$, knowledge of $p(t ; s, y, j+1)$ is needed. If and only if $\lambda_{N+1}=$ $\lambda_{N+2}=\cdots=0$ for some $N$, a backward recursion is possible, finding first $x(\cdot, \cdot, N)$ and $p(\cdot, \cdot, N)$, then $x(\cdot, \cdot, N-1)$, $p(\cdot, \cdot, N-1), x(\cdot, \cdot, N-2)$ and $p(\cdot, \cdot, N-2)$, and so on. When $x(t ; s, y, j)$ and $p(t ; s, y, j)$ are known, $u(t ; s, y, j)=$ $\widehat{u}(t, x(t ; s, y, j), p(t ; s, y, j))$.

The entities $x(t ; s, y, j)$ and $u(t ; s, y, j)$ are used to piece together a nonanticipative pair $x^{*}(t, \omega), u^{*}(t, \omega)$ defined for $t \in[0, T]$. On $\left[0, \tau_{1}\right]$, define $u^{*}(t, \omega)=u(t):=u\left(t ; 0, x_{0}, 0\right)$ and $x^{*}(t, \omega)=x(t):=x\left(t ; 0, x_{0}, 0\right)$. On $\left(\tau_{1}, \tau_{2}\right]$,

$$
\begin{aligned}
x^{*}(t, \omega) & =x\left(t, \tau_{1}, V_{1}\right) \\
& :=x\left(t ; \tau_{1}, x\left(\tau_{1}-\right)+g\left(\tau_{1}, x\left(\tau_{1}-\right), V_{1}\right), 1\right), \\
u^{*}(t, \omega) & =u\left(t, \tau_{1}, V_{1}\right) \\
& :=u\left(t ; \tau_{1}, x\left(\tau_{1}-\right)+g\left(\tau_{1}, x\left(\tau_{1}-\right), V_{1}\right), 1\right) .
\end{aligned}
$$

Generally, when $x^{*}(t, \omega)=x\left(t, \tau_{1}, V_{1}, \ldots, \tau_{j-1}, V_{j-1}\right)$ has been defined on $\left(\tau_{j-1}, \tau_{j}\right]$, then on $\left(\tau_{j}, \tau_{j+1}\right]$

$$
\begin{aligned}
x^{*}( & t, \omega) \\
= & x\left(t, \tau_{1}, V_{1}, \ldots, \tau_{j}, V_{j}\right) \\
:= & x\left(t ; \tau_{j}, x\left(\tau_{j}-, \tau_{1}, V_{1}, \ldots, \tau_{j-1}, V_{j-1}\right)\right. \\
& \left.\quad+g\left(\tau_{j}, x\left(\tau_{j}-, \tau_{1}, V_{1}, \ldots, \tau_{j-1}, V_{j-1}\right), V_{j}\right), j\right), \\
u^{*}( & t, \omega) \\
= & u\left(t, \tau_{1}, V_{1}, \ldots, \tau_{j}, V_{j}\right) \\
:= & u\left(t ; \tau_{j}, x\left(\tau_{j}-, \tau_{1}, V_{1}, \ldots, \tau_{j-1}, V_{j-1}\right)\right. \\
& \left.+g\left(\tau_{j}, x\left(\tau_{j}-, \tau_{1}, V_{1}, \ldots, \tau_{j-1}, V_{j-1}\right), V_{j}\right), j\right) .
\end{aligned}
$$

So $u^{*}(t, \omega)=\sum_{j=0}^{\infty} u\left(t, \tau_{1}, V_{1}, \ldots, \tau_{j}, V_{j}\right) 1_{\left(\tau_{j}, \tau_{j+1}\right]}(t)$ and $x^{*}(t, \omega)=\sum_{j=0}^{\infty} x\left(t, \tau_{1}, V_{1}, \ldots, \tau_{j}, V_{j}\right) 1_{\left(\tau_{j}, \tau_{j+1}\right]}(t)$ (where, for $j=0$, the $u$-and $x$-functions in the sums are simply $u(t)$ and $x(t))$. Let $p(t, \omega)=\sum_{j=0}^{\infty} p\left(t ; \tau_{j}, x^{*}\left(\tau_{j}+, \omega\right), j\right) 1_{\left(\tau_{j}, \tau_{j+1}\right]}(t)$. (These entities in fact satisfy a maximum principle not stated here: see page 126 and page 131 in [6].) (If there are restrictions of the form $x(t-, \omega) \in Q_{j}(t)$ in the problem, one only needs to construct solutions for $(s, y) \in Q_{j}(s)$, for $j>0$, for $j=0$, only for $(s, y)=\left(0, x_{0}\right)$. Eventually, one has to test if $x^{*}(t-, \omega) \in Q_{j}$ for $t \in\left(\tau_{j}, \tau_{j+1}\right.$.)

So far, we have rendered conditions pertaining to a free terminal state problem. If there are hard terminal conditions (hard = holding a.s.) or soft terminal conditions (holding in expectation), then certain transversality conditions have to be satisfied by the $p(t, \omega)$-functions; we may have to allow $H$-functions $p_{0} f_{0}+p f$, where $p_{0} \in\{0,1\}$, and for hard terminal conditions, certain controllability conditions have to 
be satisfied. Such conditions are omitted here, since our main interest is sufficient conditions; what we then need is a sort of transversality condition at the terminal time as given by (A.13), working for all types of terminal restrictions.

Assume now that entities $x(t ; s, y, j), u(t ; s, y, j)$, $p(t ; s, y, j), x^{*}(t, \omega)$, and $u^{*}(t, \omega)$ have been constructed by means of the extremal method described above, and define $\widehat{H}(t, x, p)=\sup _{u \in U} H(t, x, u, p)$. Then, the following sufficient condition holds (here $p_{0}=1$ ).

Theorem 3. If all the entities $h_{0}(x), g_{0}(t, x, V), \widehat{H}(t, x$, $p(t ; s, y, j))$, and $p(t ; s, y, j) g(t, x, V)$ are concave functions of $x$, then the control $u^{*}(t, \omega)$ is optimal in the free end case. (In case of restrictions of the form $x(t-, \omega) \in Q_{j}(t)$, again $x^{*}(t, \omega)$ must of course satisfy them, $Q_{j}(t)$ must be convex, and, for each $j$, the just-mentioned concavity needs to hold only on $Q_{j}(t)$. If $U$ is convex, then concavity of $(x, u) \rightarrow H(t, x, u, p(t ; s, y, j))$ in $Q_{j}(t) \times U$ suffices for concavity of $\widehat{H}(t, x, p(t ; s, y, j))$ in $Q_{j}(t)$.) In case one has terminal conditions on the solutions, then $u^{*}(t, \omega)$ is optimal, provided also that the following condition holds:

$$
\begin{array}{r}
E\left[p(T, \omega)\left\{x(T, \omega)-x^{*}(T, \omega)\right\}\right] \geq 0, \\
\text { for all admissible } x(\cdot, \omega),
\end{array}
$$

that is, for all $x(\cdot, \omega)$ satisfying the terminal conditions.

In the end constrained case, $p(T, \omega)$ is perhaps different from $h_{0 x}\left(x^{*}(T, \omega)\right)$. See proof of Theorem, page 30 in [7]. Note that (A.13) is implied by standard transversality conditions for soft end constraints that are omitted here; see (3.62), page 146 in [6] for the case $h_{0} \equiv 0$.

When $T=\infty$, these concavity conditions again ensure optimality (perhaps catching up optimality) provided (see [7], page 32)

$$
\begin{array}{r}
\liminf _{T \rightarrow \infty} E\left[p(T, \omega)\left\{x(T, \omega)-x^{*}(T, \omega)\right\}\right] \geq 0, \\
\text { for all admissible } x(\cdot, \omega) .
\end{array}
$$

Boundedness, Lipschitz-and growth-conditions on $u^{*}, g^{0}$, $f^{0}, f$, and $g$ are required, as in Theorem 2 .

\section{Acknowledgment}

The author is very grateful for useful comments received from a referee that made it possible to improve the exposition and remove some typographical errors.

\section{References}

[1] N. Bauerle and U. Rieder, Markov Decision Processes with Applications to Finance, Springer, New York, NY, USA, 2011.

[2] M. H. A. Davis, Markov Models and Optimization, Chapman \& Hall, London, UK, 1992.

[3] N. Bäuerle and U. Rieder, "MDP algorithms for portfolio optimization problems in pure jump markets," Finance and Stochastics, vol. 13, no. 4, pp. 591-611, 2009.
[4] M. Schäl, "On piecewise deterministic Markov control processes: control of jumps and of risk processes in insurance," Insurance: Mathematics and Economics, vol. 22, no. 1, pp. 75-91, 1998.

[5] B. Øksendal and A. Sulem, Applied Stochastic Control of Jump Diffusions, Springer, Berlin, Germany, 2005.

[6] A. Seierstad, Stochastic Control in Discrete and Continuous Time, Springer, New York, NY, USA, 2009.

[7] A. Seierstad, "Necessary conditions and sufficient conditions for optimal control of piecewise deterministic control systems," Memorandum No 5/2001, Department of Economics, University of Oslo, 2001. 


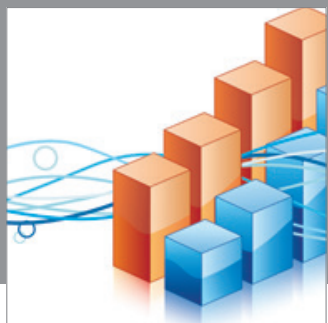

Advances in

Operations Research

mansans

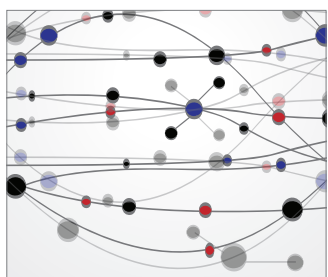

The Scientific World Journal
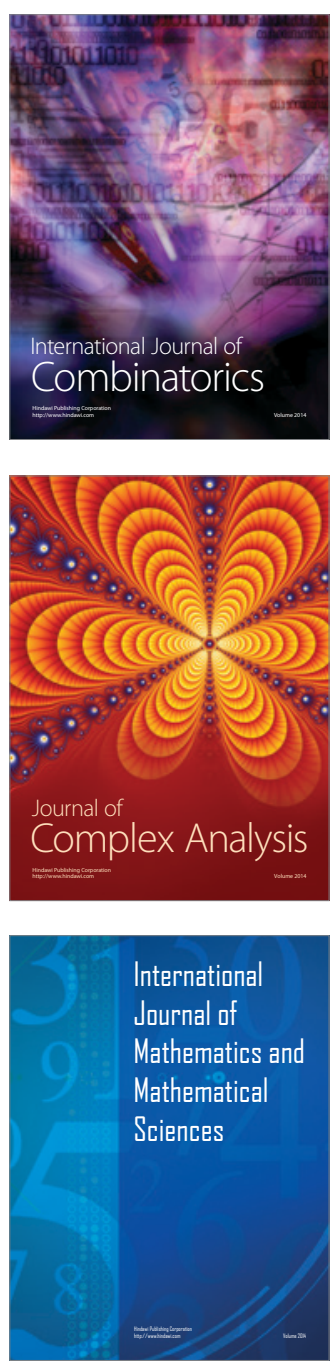
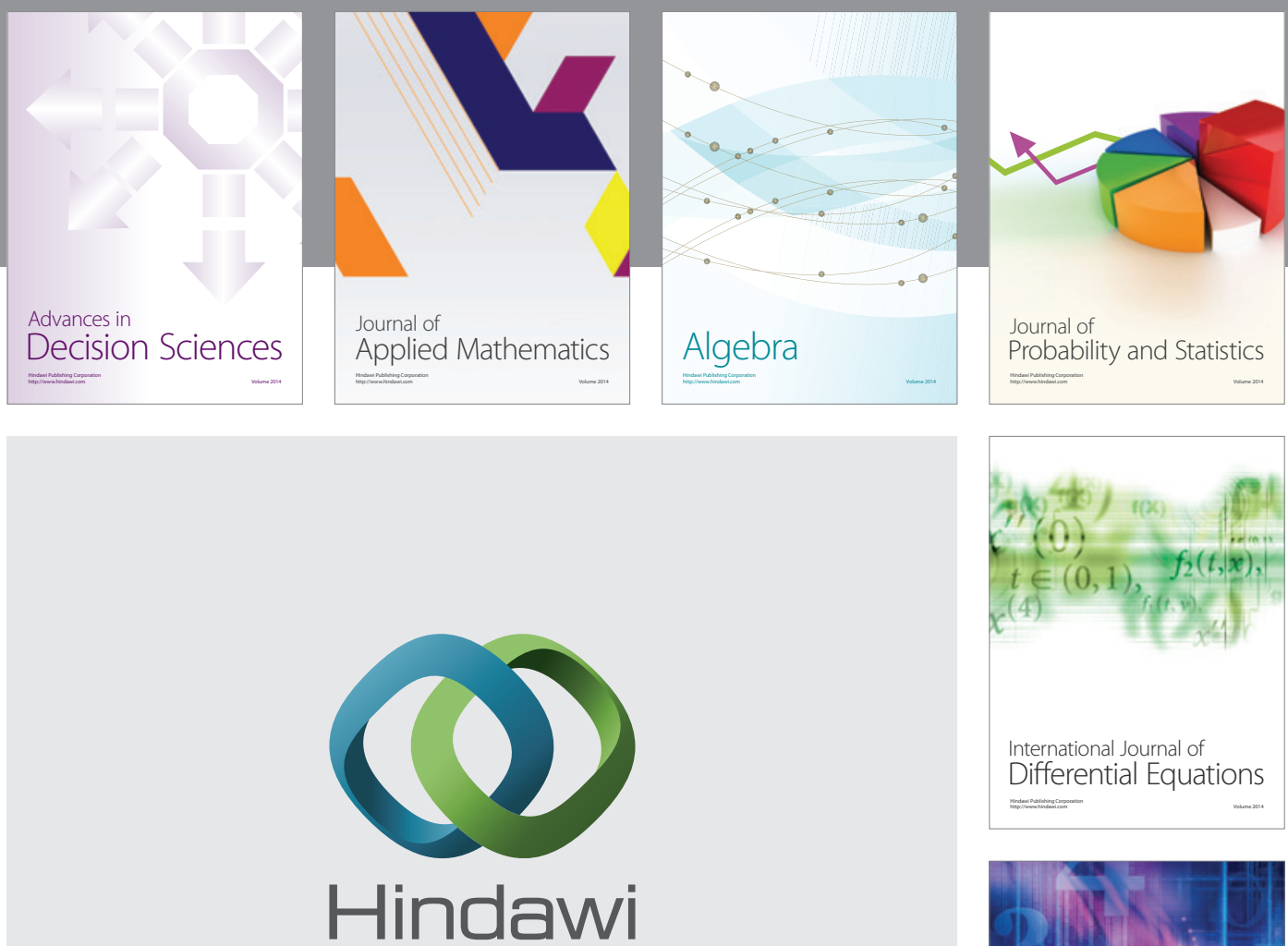

Submit your manuscripts at http://www.hindawi.com
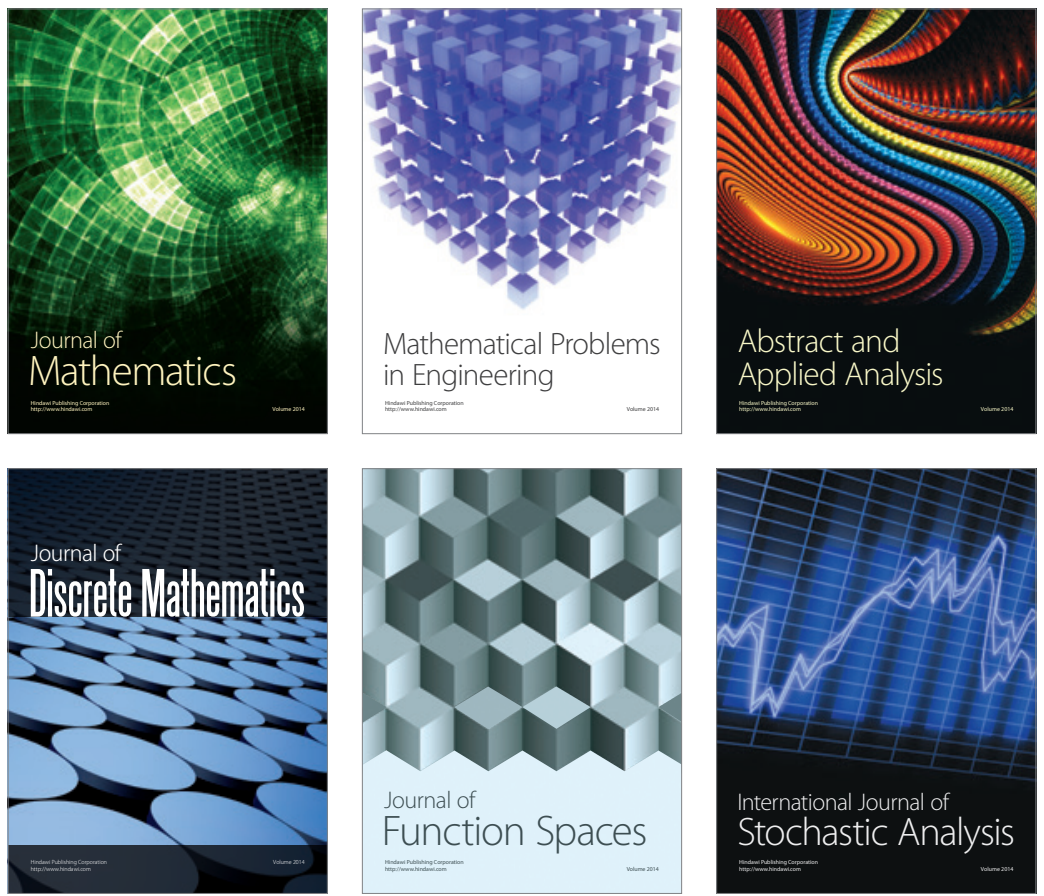

Journal of

Function Spaces

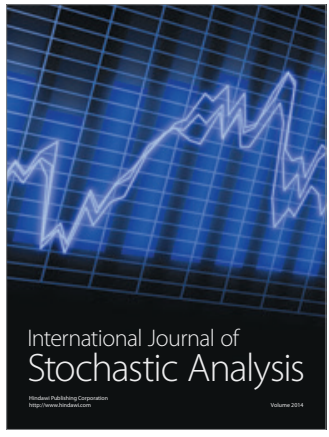

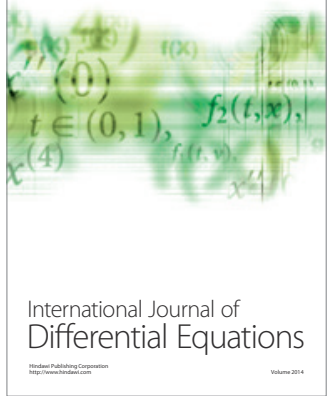
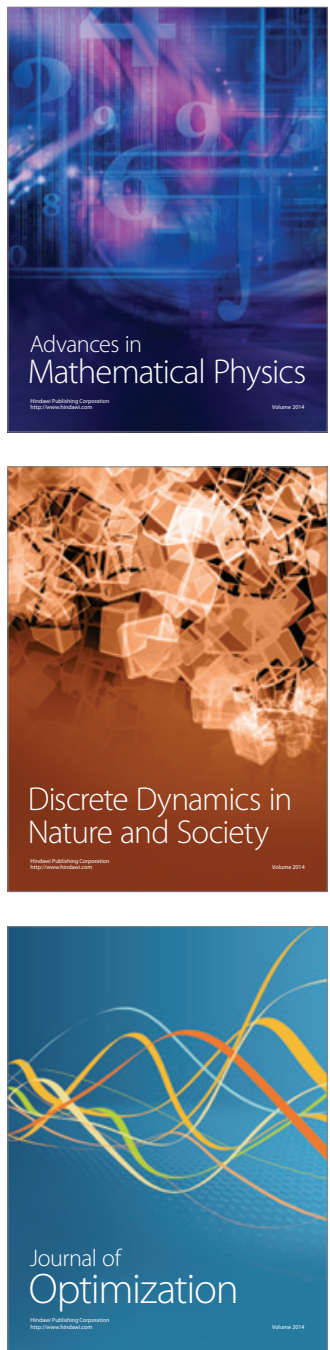